\title{
Providing Real-time Feedback for Student Teachers in a Virtual Rehearsal Environment
}

\author{
Roghayeh Barmaki \\ Computer Science Division \\ University of Central Florida \\ Orlando, FL, USA \\ barmaki@cs.ucf.edu
}

\author{
Charles E. Hughes \\ Computer Science Division \\ University of Central Florida \\ Orlando, FL, USA \\ ceh@cs.ucf.edu
}

\begin{abstract}
Research in learning analytics and educational data mining has recently become prominent in the fields of computer science and education. Most scholars in the field emphasize student learning and student data analytics; however, it is also important to focus on teaching analytics and teacher preparation because of their key roles in student learning, especially in K-12 learning environments. Nonverbal communication strategies play an important role in successful interpersonal communication of teachers with their students. In order to assist novice or practicing teachers with exhibiting open and affirmative nonverbal cues in their classrooms, we have designed a multimodal teaching platform with provisions for online feedback. We used an interactive teaching rehearsal software, TeachLivE ${ }^{\mathrm{TM}}$, as our basic research environment. TeachLivE employs a digital puppetry paradigm as its core technology. Individuals walk into this virtual environment and interact with virtual students displayed on a large screen. They can practice classroom management, pedagogy and content delivery skills with a teaching plan in the TeachLivE environment.
\end{abstract}

We have designed an experiment to evaluate the impact of an online nonverbal feedback application. In this experiment, different types of multimodal data have been collected during two experimental settings. These data include talk-time and nonverbal behaviors of the virtual students, captured in log files; talk time and full body tracking data of the participant; and video recording of the virtual classroom with the participant. 34 student teachers participated in this 30 minute experiment. In each of the settings, the participants were provided with teaching plans from which they taught. All the participants took part in both of the experimental settings. In order to have a balanced experiment design, half of the participants received nonverbal online feedback in their first session and the other half received this feedback in the second session. A visual indication was used for feedback each time the participant exhibited a closed, defensive posture. Based on recorded full-body tracking

Permission to make digital or hard copies of all or part of this work for personal or classroom use is granted without fee provided that copies are not made or distributed for profit or commercial advantage and that copies bear this notice and the full citation on the first page. Copyrights for components of this work owned by others than ACM must be honored. Abstracting with credit is permitted. To copy otherwise, or republish, to post on servers or to redistribute to lists, requires prior specific permission and/or a fee. Request permissions from Permissions@acm.org.

ICMI '15, November 09-13, 2015, Seattle, WA, USA

(C) 2015 ACM. ISBN 978-1-4503-3912-4/15/11 ... \$15.00

DOI: http://dx.doi.org/10.1145/2818346.2830604 data, we observed that only those who received feedback in their first session demonstrated a significant number of open postures in the session containing no feedback. However, the post-questionnaire information indicated that all participants were more mindful of their body postures while teaching after they had participated in the study.

\section{Categories and Subject Descriptors}

H.5.1. [Information Interfaces and Presentation (e.g. HCI)]: Multimedia Information Systems

\section{Keywords}

Immersive teaching rehearsal environment; Teacher training; Posture recognition; Mutimodal data analytics;

\section{INTRODUCTION}

Communication involves both verbal and nonverbal components. Nonverbal communication (NVC) is all of the elements of communication excluding the words [20]. Paralanguage (pitch, volume, etc.) is the vocal element of nonverbal communication. On the other hand, body language (gestures and movements, facial expressions, eye contact, etc.) is the nonvocal element of nonverbal communication [16]. Upwards of 65 percent of the meaning derived in interactions comes from nonverbal communication [20,11].

Gestures, the primary focus of this research, are a form of nonverbal communication in which visible body movements and poses are used to communicate important messages, either in place of speech or together and in parallel with spoken words [15]. The context in which we consider gestures is the student-teacher interaction that occurs in a classroom setting. Here, NVC is known to play a central role in the successful interaction that occurs between student and teacher [6]. The research indicates that teachers' gestures have an impact, both positive and negative, on student comprehension and student learning, specifically in instructional discourses $[29,21]$. Thus, to be effective in the classroom, teachers must not only be aware of their own nonverbal cues but also must be cognizant of those being transmitted by the students [6].

This paper reports the results of an investigation of the nonverbal behaviors (body movements, especially gestures and postures) of participants in an immersive rehearsal environment. In this experiment different modalities have been captured, such as event-logs and video captures from participant 
and virtual classroom; however the main focus was to perceive the relations of a participant's teaching experience with body posture feedback exhibited in a simulated classroom. In addition, the participants were asked to take pre and post questionnaires during the experiment to determine their perception of the experience. The eventual goal of the research is to design a semi-automated annotation system for body language and gesture tracking of participants in the virtual classroom environment that leads to multimodal assessment and reflection procedures for professional development.

\section{BACKGROUND AND RELATED WORK}

\subsection{The Teaching Rehearsal Environment}

Our test-bed environment, TeachLivE, is a teaching and learning environment that was developed to provide an interactive learning experience for teachers to rehearse and hone their classroom skills. The environment uses a virtual classroom setting that is typically inhabited by five or six virtual students, commonly called avatars. Each virtual student has behaviors that reflect their personalities and backstories. A single human, called an interactor, uses a combination of agency and digital puppeteering to orchestrate the behaviors of all students in a manner that reflects how each would react to the teacher participant's actions. Figure 1 depicts the core architecture of the TeachLivE system [22], with some features that have been added to support the research reported here.

In the TeachLivE paradigm, users of the system, typically university faculty members or school district coaches, specify subject matter, a teaching plan, student behaviors (e.g., cooperative, needing encouragement, terribly distracted) and other desired attributes at least two weeks in advance of a planned use of the environment.

Over its almost decade of evolution, TeachLivE has gone from a local resource to a system used at over 55 universities in the training of some 12,000 teacher candidates during the 2014-15 academic year. In addition to its use as a pedagogical tool, partnering universities have carried out significant research projects within the TeachLivE environment. One of the largest of these studies was funded by the Bill and Melinda Gates Foundation. This study involved approximately 150 in-service teachers and demonstrated that four ten-minute sessions in the simulated classroom were sufficient to charge a small set of targeted behaviors that have been shown to be critical elements of effective teaching [8].

In one of our previous formative studies [4], we used an after-action review tool-set as an interface for manual annotation of the nonverbal behaviors (body language (open versus closed poses) and head movement (nodding)) for a group of teachers. That data, along with automated recording of proximity and talk time (teacher versus students) were used as a first step (proof of concept) in our research plan. The currently reported research employs the Microsoft Kinect V2 SDK and 3D-sensor technology [2] to automate the nonverbal communication coding procedure. We discuss details of the modality and features of the recording process in the method section.

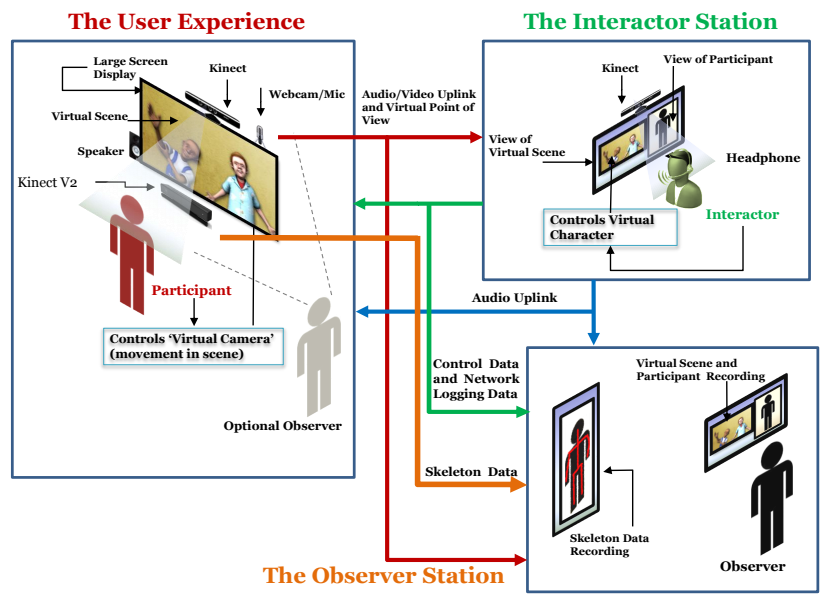

Figure 1: The test-bed environment with some adjustments from the original architecture. The recorded streams are: (I) skeleton data (IR, depth and body frame) shown with orange arrow and (II) virtual scene paired with user experience recording from the observer station (video stream) with red arrow and (III) network logging data including virtual classroom's verbal and nonverbal behaviors with green arrow.

\subsection{Nonverbal Communication}

Nonverbal communication has been divided into nine categories: kinesics, more commonly referred to as body language and physical appearance; chronemics or communication through time; proxemics or communication using space; para-language, communication through tone of voice; artifacts, communication by physical objects; haptics, communication through touch; facial expressions; olfactics, communication by means of smells; and oculesics, more commonly referred to as eye contact [27]. The presented research focuses on kinesics and body language, especially gestures from defined NVC categories.

McNeill categorizes gestures into four main types [19]. Iconics are related to semantic content of speech; metaphorics tie to an abstract concept; deictics or pointing gestures relate to the identity of individuals to whom the utterances refer; and beats keep the rhythm of speech [13, 19].

NVC plays an important role in several professions. In the case of physician-patient interaction, scholars presented results on the relation of nonverbal communication skill of the physicians and the patient satisfaction $[7,9]$. In the classroom environment, the successful student-teacher communication is also affected by the NVC skills of the teacher $[17,6]$.

\subsubsection{Nonverbal Communication in Immersive Envi- ronments}

There is extensive research on the impact and application of gestures in virtual and interactive environments. The context of deictic expressions and the impact of pointing gestures in virtual immersive environment have been investigated in [26]. Two participants were asked to play an object 
identification game together and the session was recorded and annotated based on gaze finger pointing and index-finger pointing to analyze the success rate of object identification. In a similar study [10] from the same research lab the applications of iconic gestures to express shape-related references to objects in a virtual construction environment were investigated. They showed how to make use of the Imagistic Description Tree (IDT) formalism to enhance virtual construction parts with shape-related information.

In [12], the scholars introduced an automated system for social skills training called MACH (My Automated Conversation $\mathrm{CoacH}$ ). The system includes a virtual agent that reads facial expressions, speech, and prosody (tone, pitch) of the participant and responds with verbal and nonverbal behaviors in real-time. As an example application, the developers of $\mathrm{MACH}$ created an interview trainer that asks questions appropriate for a job interview, automatically mimicking certain behaviors displayed by the user, and exhibiting appropriate nonverbal behaviors [12].

\subsubsection{Nonverbal Communication in Classroom}

Nonverbal communication strategies are consistently noted in successful approaches to teacher training [18]. The effects of strategies like eye contact, prolonged gaze, and proximity can have positive or negative effects on student behavior and classroom management, depending on the situation and context [17].

Teachers can use gesture to be effective in several fundamental aspects of their profession, including communication, assessment of student knowledge, and the ability to instill a profound understanding of abstract concepts in traditionally difficult domains such as language and mathematics [13].

There is a significant body of research on the impact of gestures in the classroom. Kelly et al. showed that employing iconic gestures during teaching had a significant impact on English-speaking adults attempting to learn new Japanese words [14]. In a recent study from Wang and Loewen [31] the employment of nonverbal behaviors of teachers as feedback in second language acquisition was investigated. They explored teachers' nonverbal behaviors in corrective feedback during 48 observations (about 65 hours of recordings) of nine classrooms for English as a second language. The results indicated that teachers used a variety of nonverbal behaviors in their corrective feedback, including hand gestures (specifically iconics, metaphorics, deictics, and beats), head movements, affect displays, kinetographs, and emblems.

Alibali et al. [3] observed that teachers use gestures in an effort to scaffold student's understanding, especially on abstract concepts. In this research, they worked on a 20minute sixth-grade mathematics lesson session that focused on algebraic relations [3]. They manually annotated the gestures and transcribed the teacher's conversation in the study and used the annotation data to analyze the nonverbal behavior of the teacher.

In [5], we investigated the way teachers use gestures in the virtual classroom settings of TeachLivE. Our research had been developed based on the hypothesis reported in [3]. Biology and algebra teachers were evaluated in this study.
Analysis of video recordings from real and virtual classroom environments indicated that algebra teachers gesture significantly more often than biology teachers [5].

\subsubsection{Multimodal Discourse Analysis (MDA)}

Employing a multimodal approach to classroom analysis has implications for teaching and learning as well as teacher training and development. Multimodal discourse analysis (MDA) is an emerging paradigm in discourse studies which extends the study of language per se to the study of language in combination with other resources, such as images, scientific symbolism, gesture, action, music and sound [25]. In a project done in a classroom in Singapore [24], the meanings made in the multimodal pedagogic discourse, specifically in language, gesture, positioning and movement were investigated. Their project particularly focused on proxemics to multimodal classroom discourse analysis as well as material distance socio-semiotic meanings.

\subsubsection{Nonverbal Behavior Annotation}

Manual annotation of nonverbal behaviors using the video recordings by experts can provide accurate annotations; however it is extremely time consuming [23, 28]. Hence, some automated techniques were presented for gesture/posture recognition from video clips and other resources. In general, most of gesture recognition engines have a set of training data to learn and extract the patterns of the gesture from the training set and then use the trained engine for recognition. In this research, we use a similar technique with skeleton data in the Visual Gesture Builder environment [2]. This approach is fast and inexpensive in comparison to the hand-coded procedure.

\section{METHOD AND MATERIALS}

\subsection{Recording Specifications}

Different types of data were recorded during the experiment. (I) full-body tracking data (IR, depth and body frame), (II) virtual scene paired with user experience from the observer station (video recording) and finally (III) network logging data including virtual students' verbal (talk-time) and their nonverbal behaviors in the experiment session. Figure 1 presents the room setting and data collection specifications. The skeletal data of the participants were recorded using the Kinect V2 sensor and Kinect Studio utility application. The recorded clips with the Kinect Studio are required as data inputs to Visual Gesture Builder (VGB) to do automated tagging of gestures [2]. Further details are presented in the following subsection.

The second type of recorded data was the virtual classroom scene (from the observer workstation) paired with user experience (participant's front view as seen through the Skype application) in mp4 format that was recorded with a window recording application-Bandicam. Lastly, the network traffic data was recorded to keep track of interactions of the virtual characters. Virtual avatars' NVC signals and their talk-time were recorded in a log-file with comma-delimited format in an observer station for future multimodal analysis such as revealing the correlations of the teacher's wait-time with the student's talk-time or the teacher's talk-time with teacher's body movement data in the two settings. 


\subsubsection{VGB Automated Tagging and its Input Corpus}

VGB generates gesture databases that are used by applications to perform real-time gesture detection. In the technical document reported by Microsoft team [2], VGB is defined as a data driven machine learning solution for gesture detection. Visual Gesture Builder uses skeleton tracking data (25 body points in Kinect V2) representing body movements, and AdaBoost ensemble classifier (for discrete gestures) or Random Forest Regression (for continuous gestures).

First of all, for each gesture, some clips from different subjects must be recorded with Kinect Studio. Every clip contains an individual facing the Kinect (and within the Kinect's field of view) who exhibits the gesture (with some variations) 5 to 15 times. Then, the VGB tool is used to give meaning to the recorded clips. All the frames in the recordings that define a gesture, must be tagged manually. For each gesture, a project as a binary classifier is trained. All the frames exhibiting the gesture get the label of 1 and the remaining frames in the clip get the value of -1 .

At this point, the problem is similar to a supervised binary classification, in which the classifier will recognize the frames in which a gesture is performed. Depending on the number of gestures that we intend to recognize, the gesture recognition engine will have binary classifiers. After the training step, VGB is able to generate the tags automatically in the analysis step from the test clips. In the following subsection we discuss more details about targeted gestures to recognize according to interpersonal communication perspective.
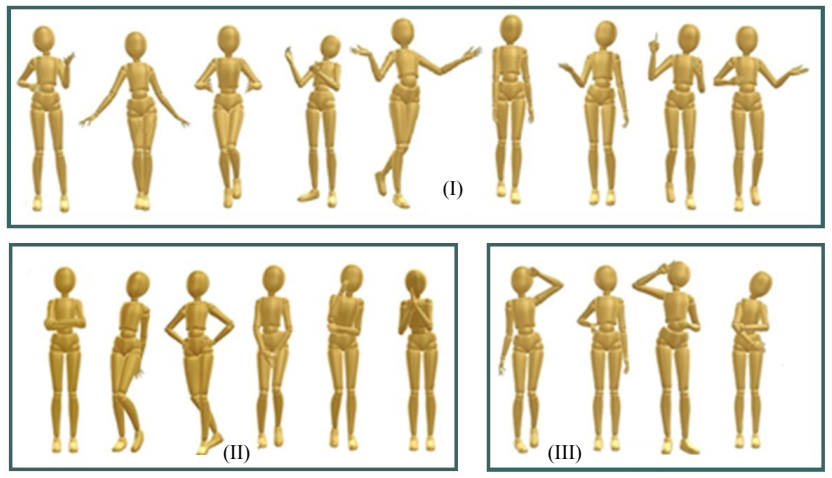

Figure 2: Reference example of (I) open poses, (II) closed poses; left to right: unreceptive (arms folded in front), seductive (hands clasped in back) skeptical (hands placed on hips) protective (hands clasped in lower front) pensive (thoughtful pose) submissive (hands clasped in upper front), and (III) ambivalent poses.

Some of the most common and interesting postures from the teaching evaluation literature [6] and from our previous study [4] are shown in Figure 2. Caswell and Neill in [6] present results on body language of competent teachers. Using reference poses as a communication medium, they recommend that teachers employ open rather than closed or ambivalent poses in their classroom. Closed poses indicate defensiveness and avoidance by the speaker/teacher of the listener [30]. In addition, ambivalent poses, which are mainly a combination of both closed and open poses, cause confusion and misconception about the speaker's behavior and intention in the listeners'/students' mind.

A corpus of closed body poses (reference poses in figure 2.II and some variations of them) was recorded from the performances of three male and two female students using the Microsoft Kinect V2. Subjects for the main study were not involved in the corpus collection. The corpus was used as training clips in the Visual Gesture Builder (VGB) application. All the clips were hand-coded and tagged in order to feed the automated analysis procedure of the participant's clips. There were six targeted closed gestures/postures to train/evaluate in this research. For training each posture, some 30-sec clips were manually annotated and added to the gesture database. $7 \times 5$ short clips were manually annotated in total since the thinking or pensive gesture is a sided-gesture, and we needed to record left and right versions for that gesture. Hence, we had seven classifiers rather than six at the end of training for the initial six gestures. The process of creating the gesture recognition engine [2] is shown in figure 3 .

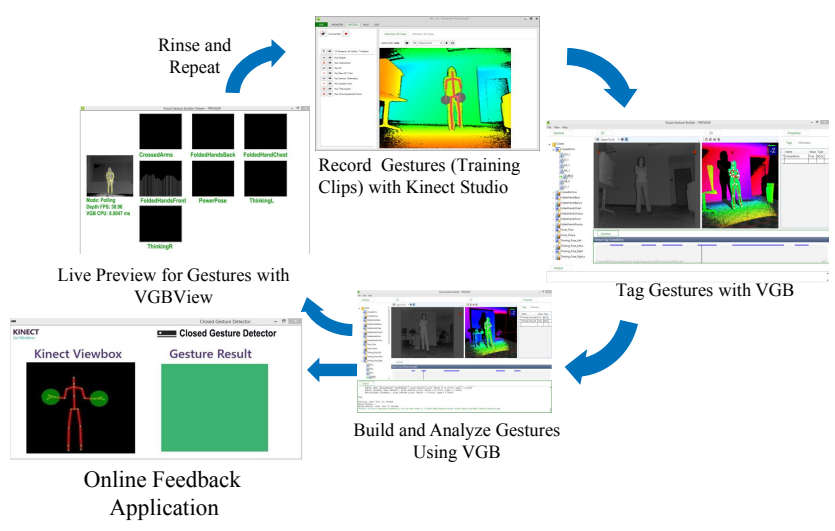

Figure 3: The data-driven and incremental process of creating a gesture detector using the Microsoft SDK 2.0 and the VGB utility[2]. The proposed online feedback application is developed on top of the gesture detector database.

\subsection{Recruitment Procedure}

In this experiment, 34 student participants were recruited. Only those College of Education students who had seen the TeachLivE environment before the experiment were eligible to be recruited. Participants were ones over age 18 years from the affiliated university community and each participant had a basic level of English proficiency, with some teaching experience to be able to talk and interact with the virtual students in the TeachLivE.

All participants were from the students who were registered for a classroom management and strategies class in the TESOL (Teaching English to Speakers of Other Languages) program taught in the summer semester at the College of Education. All the students had an assignment prior to the experiment using the TeachLivE environment. The experiment was mandatory for all of the registered students as a classroom activity because of its perfect overlap with the course goals. Students did not get compensation or credit for their participation. Individuals were expected to spend 
approximately 30 minutes for the experiment (two 7-minute sessions for the teaching plus three 5-minutes intervals for questionnaires).

\subsection{Design Details}

The experiment was designed to have two treatments: with and without feedback provision to participants in the virtual classroom session (7-minutes long each). Feedback was in the form of a visual indication each time the participant exhibits a closed stance while interacting with the virtual students. Figure 4 indicates the multiple settings for the experiment. The feedback application is designed based on an existing closed body posture corpus and a new interface for automated online (real-time) feedback. All the participants experienced both of the treatments. The experiment is designed based on $2 \times 2$ Latin-squares in order to balance the experimental design; therefore half the participants received real-time feedback (treatment or setting 2) during this first session while the other half received no feedback (setting 1) during this session.

Participants were given a consent form, a pre-questionnaire and a teaching plan before their first experience. After completing the pre-questionnaire and reviewing the teaching plan and consent form, the participants taught their first session. Afterwards, all participants filled out a postquestionnaire. They then reentered the virtual classroom to present the same teaching plan and had their second treatment accordingly. All participants were then asked to complete the same post-questionnaire that they filled out after the first session.

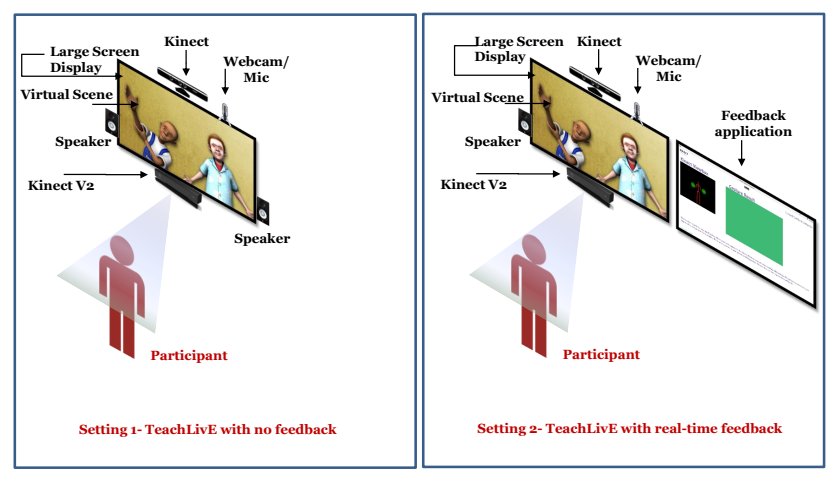

Figure 4: The settings of the experiment.

\subsubsection{Questionnaires}

This experiment had one pre-questionnaire and one postquestionnaire. In the pre-questionnaire, participants were asked to indicate their age, gender, ethnicity, major and highest level of education, teaching experience and related teaching topics, and finally their particular experience with the TeachLivE environment.

The post-questionnaire was designed based on the multimodal learning application evaluation and design guide with some changes ${ }^{1}$. There were different types of questions about naturalness of use, boredom, intrusiveness, likelihood of use

\footnotetext{
${ }^{1}$ http://sigmla.org/mla2015/ApplicationGuidelines.pdf accessed on August 2015.
}

during spare time, learning experience, comparison with traditional learning methods, the novelty of the system and some open-ended questions. Based on the different nature of our experiment (teacher evaluation rather than student evaluation), there were some questions in the original questionnaire that were not applicable to our experiment, therefore we removed them from our post questionnaire. We also modified some of the questions slightly to match our proposed application.

\subsubsection{Teaching Plan}

The teaching plan we employed is designed to enhance science literacy and is aligned with disciplinary core ideas and cross-cutting concepts from the Next Generation Science Standards ${ }^{2}$, as well as the Common Core Standards for Literacy in Science ${ }^{3}$. The lesson is based on the $5 \mathrm{E}$ instructional model and has been validated and field-tested in high school Biology classrooms as part of a larger module from the NIH Curriculum Supplement Series "Using Technology to Study Cellular and Molecular Biology"[1]. The title of the teaching plan was: "What is Technology?" In this teaching plan, some questions were mentioned to discuss with virtual students by the participant. The participant, as the teacher, was expected to ask virtual students about their personal definition of technology, what does technology do for human beings, what technologies are available in the room, and the impact of technology in diseases. The participants were free to discuss any concept they preferred within the topic of this teaching plan.

\subsubsection{Room Setting}

The room was equipped with required laptops and wired network connection for the experiment. Three laptops were used for the experiment: one as an observer workstation, one for skeletal data recording and visualized feedback, and the last one for the participant's workstation. Figure 1 and figure 4 present further details about the room setting. The virtual classroom's students were high-school avatars (three males and two females with their own predefined characteristics). Their level of misbehavior was set to zero which means no misbehavior in the classroom-side. We also scheduled the same interactor for all the experiment sessions in order to minimize the confounding conditions related to the inter-actor change.

Next to the participant's large TV display, we posted all the teaching plan's main questions on a board as reference for participants. This was a very important step, since all of the participants requested to keep this sheet of paper in their hands during the interaction. We asked them to leave the teaching plan and use the reference board instead. This was critical as the gestures of participants were affected negatively while they were holding an object (even a sheet of paper) in their hands.

\subsubsection{Feedback Application}

As mentioned before, feedback was in the form of a visual indication each time the participant exhibited a closed, defensive stance. We used the Kinect V2 and the closed-postures

\footnotetext{
${ }^{2}$ http://www.nextgenscience.org accessed on August 2015.

${ }^{3}$ http://www.corestandards.org/ELA-Literacy/RST accessed on August 2015.
} 
corpus as input to this application. The feedback application ran independently from the TeachLivE program.

Figure 5 presents the feedback user interface in more details than figure 4. Most of the participants changed their body movements in the feedback setting to prevent the orange box from showing.

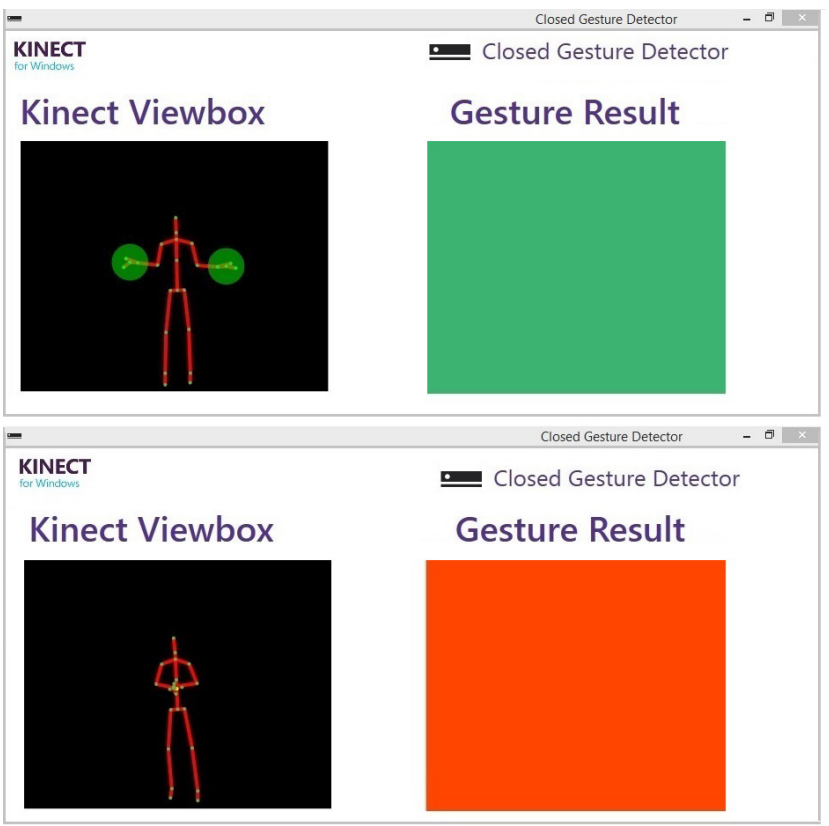

Figure 5: The feedback application user-interface for two different body postures. The user can see his/her body posture in real-time in the Kinect ViewBox. Whenever the participant exhibits one of the closed gestures that were targeted, the Gesture Result window become orange from green automatically.

\subsection{Preliminary Observations}

As might be expected, those who received body posture feedback in their first session displayed more awareness of their nonverbal messaging (significant employment of open postures) in the second session. In contrast, those who received no feedback in first session showed only slight improvement in the second, despite the availability of real-time feedback. We hypothesize that this small improvement came from their increased comfort with the TeachLivE environment. Interestingly, members of both groups indicated in the post-questionnaire that they had gained an awareness of the messages they were sending through their body poses and expressed the intent to employ these strategies in their teaching.

In addition, there was a difference between experienced teachers and novice teachers in their body language. We observed that experienced teachers did not exhibit closed gestures as often as students who had no teaching experience. Also, among the detected gestures, submissive and seductive gestures were the most frequent closed gestures exhibited by participants.

\section{FUTURE STEPS}

We intend to complete the data analysis and reporting the correlations from the multimodal assessment point-of-view. This phase involves multimodal signal processing: data synchronization, data fusion and data mining.

Recruiting the participants who already had this experience after one-semester (or later) and observing their potential body movement changes is another interesting direction to follow, since most of the participants were very motivated to experience the environment one more time. In addition, most of the participants indicated valuable information in their post-questionnaire about how to improve the application. For example, most of the students requested to have a more immersive experience. This might be done by using Head-Mounted-Displays (HMD) for instance, although HMDs introduce an additional confound by changing people's sense of being in a physical environment.

\subsection{Acknowledgments}

The authors wish to thank Drs. Lisa Dieker, Michael Hynes, Cynthia Hutchinson, Joyce Nutta, Thomas Owens, Jacqueline Rodriguez, Guo-Jun Qi, Carrie Straub, and the entire TeachLivE team including Pam Jones and Michael Hopper for their support during the data collection and analysis processes. Their insights and their assistance with subject recruitment were invaluable. We also wish to express our appreciation for the support of the interactors and the study participants. Finally we wish to acknowledge support for the evolution of the TeachLivE project from the Bill \& Melinda Gates Foundation (OPP1053202) and the National Science Foundation (CNS1051067, IIS1116615). Any opinions, findings, and conclusions or recommendations expressed in this material are those of the authors and do not necessarily reflect the views of the sponsors.

\section{REFERENCES}

[1] Using technology to study cellular and molecular biology. http://1.usa.gov/1LKos30. Accessed 2015-8-5.

[2] Visual gesture builder: A data-driven solution to gesture detection. http://aka.ms/k4wv2vgb, July 2014. Accessed 2015-8-8.

[3] M. W. Alibali and M. J. Nathan. Teachers' gestures as a means of scaffolding students' understanding: Evidence from an early algebra lesson. Video research in the learning sciences, pages 349-365, 2007.

[4] R. Barmaki. Nonverbal communication and teaching performance. In Proceedings of the Eighth International Conference on Educational Data Mining, EDM '14, pages 441-443, 2014.

[5] R. Barmaki and C. E. Hughes. A case study to track teacher gestures and performance in a virtual learning environment. In Proceedings of the Fifth International Conference on Learning Analytics And Knowledge, LAK '15, pages 420-421, New York, USA, 2015.

[6] C. Caswell and S. Neill. Body language for competent teachers. Routledge, 2003.

[7] L. G. Collins, A. Schrimmer, J. Diamond, and J. Burke. Evaluating verbal and non-verbal communication skills, in an ethnogeriatric osce. Patient education and counseling, 83(2):158-162, 2011.

[8] L. A. Dieker, C. L. Straub, C. E. Hughes, M. C. 
Hynes, and S. Hardin. Learning from virtual students. Educational Leadership, 71(8):54-58, 2014.

[9] M. R. DiMatteo, R. D. Hays, and L. M. Prince. Relationship of physicians' nonverbal communication skill to patient satisfaction, appointment noncompliance, and physician workload. Health Psychology, 5(6):581, 1986.

[10] C. Fröhlich, P. Biermann, M. E. Latoschik, and I. Wachsmuth. Processing iconic gestures in a multimodal virtual construction environment. In Gesture-Based Human-Computer Interaction and Simulation, pages 187-192. Springer, 2009.

[11] L. K. Guerrero and K. Floyd. Nonverbal communication in close relationships. Routledge, 2006.

[12] M. E. Hoque, M. Courgeon, J.-C. Martin, B. Mutlu, and R. W. Picard. Mach: My automated conversation coach. In Proceedings of the 2013 ACM international joint conference on Pervasive and ubiquitous computing, pages 697-706. ACM, 2013.

[13] S. D. Kelly, S. M. Manning, and S. Rodak. Gesture gives a hand to language and learning: Perspectives from cognitive neuroscience, developmental psychology and education. Language and Linguistics Compass, 2(4):569-588, 2008.

[14] S. D. Kelly, T. McDevitt, and M. Esch. Brief training with co-speech gesture lends a hand to word learning in a foreign language. Language and Cognitive Processes, 24(2):313-334, 2009.

[15] A. Kendon. Gesture: Visible action as utterance. Cambridge University Press, 2004.

[16] M. Knapp, J. Hall, and T. Horgan. Nonverbal communication in human interaction. Cengage Learning, 2013.

[17] R. Laslett and C. Smith. Effective classroom management: a teacher's guide. Routledge, 2002.

[18] J. Mahon, B. Bryant, B. Brown, and M. Kim. Using second life to enhance classroom management practice in teacher education. Educational Media International, 47(2):121-134, 2010.

[19] D. McNeill. Hand and mind: What gestures reveal about thought. University of Chicago Press, 1992.

[20] A. Mehrabian. Communication without words.
Psychological today, 2:53-55, 1968.

[21] P. W. Miller. Body language in the classroom. Techniques: Connecting Education and Careers, 8:28-30, 2005.

[22] A. Nagendran, R. Pillat, A. Kavanaugh, G. Welch, and C. Hughes. A unified framework for individualized avatar-based interactions. Presence: Teleoperators and Virtual Environments, 23(2):109-132, 2014.

[23] L.-V. Nguyen-Dinh, A. Calatroni, and G. Tröster. Robust online gesture recognition with crowdsourced annotations. The Journal of Machine Learning Research, 15(1):3187-3220, 2014.

[24] K. L. O'Halloran. Multimodal analysis and digital technology. In Interdisciplinary Perspectives on Multimodality: Theory and Practice, Proceedings of the Third International Conference on Multimodality, Palladino, Campobasso, 2009.

[25] K. L. O'Halloran. Multimodal discourse analysis. Companion to discourse, pages 120-137, 2011.

[26] T. Pfeiffer, M. E. Latoschik, and I. Wachsmuth. Conversational pointing gestures for virtual reality interaction: implications from an empirical study. In Virtual Reality Conference, 2008. VR'08. IEEE, pages 281-282. IEEE, 2008.

[27] V. P. Richmond, J. C. McCroskey, and M. L. Hickson III. Nonverbal behavior in interpersonal relations. 2014.

[28] D. Roggen, A. Calatroni, M. Rossi, T. Holleczek, K. Forster, G. Troster, P. Lukowicz, D. Bannach, G. Pirkl, A. Ferscha, et al. Collecting complex activity datasets in highly rich networked sensor environments. In Networked Sensing Systems (INSS), pages 233-240. IEEE, 2010.

[29] H. A. Smith. Nonverbal communication in teaching. Review of Educational Research, 49(4):631-672, 1979.

[30] J. S. Tucker and S. L. Anders. Adult attachment style and nonverbal closeness in dating couples. Journal of Nonverbal Behavior, 22(2):109-124, 1998.

[31] W. Wang and S. Loewen. Nonverbal behavior and corrective feedback in nine esl university-level classrooms. Language Teaching Research, pages 1-20, 2015. 